\title{
Raman-induced Kerr-effect dual-comb spectroscopy
}

\author{
T. Ideguchi, ${ }^{1}$ B. Bernhardt, ${ }^{1,2}$ G. Guelachvili, ${ }^{3}$ T. W. Hänsch,,${ }^{1,2}$ and N. Picqué ${ }^{1,2,3, *}$ \\ ${ }^{1}$ Max-Planck-Institut für Quantenoptik, Hans-Kopfermann-Strasse 1, Garching 85748, Germany \\ ${ }^{2}$ Ludwig-Maximilians-Universitüt München, Fakultät für Physik, Schellingstrasse 4/III, München 80799, Germany \\ ${ }^{3}$ Institut des Sciences Moléculaires d'Orsay, CNRS, Bâtiment 350, Université Paris-Sud, Orsay 91405, France \\ ${ }^{*}$ Corresponding author: nathalie.picque@mpq.mpg.de
}

Received August 7, 2012; revised September 21, 2012; accepted September 22, 2012;

posted September 25, 2012 (Doc. ID 173924); published October 26, 2012

\begin{abstract}
We report on the first (to our knowledge) demonstration of nonlinear dual-frequency-comb spectroscopy. In multi-heterodyne femtosecond Raman-induced Kerr-effect spectroscopy, the Raman gain resulting from the coherent excitation of molecular vibrations by a spectrally narrow pump is imprinted onto the femtosecond laser frequency comb probe spectrum. The birefringence signal induced by the nonlinear interaction of these beams and the sample is heterodyned against a frequency comb local oscillator with a repetition frequency slightly different from that of the comb probe. Such time-domain interference provides multiplex access to the phase and amplitude Raman spectra over a broad spectral bandwidth within a short measurement time. @ 2012 Optical Society of America

OCIS codes: $320.7090,300.6450,300.6300$.
\end{abstract}

Optical frequency combs [1]], initially developed for frequency metrology, are becoming enabling tools for a variety of applications in science and technology, including broad spectral bandwidth molecular spectroscopy. New comb-based approaches to molecular spectroscopy are so far only probing the absorption and dispersion of the samples, mostly in the gas phase. Among these, dualcomb spectroscopy [2-6] holds much promise for rapid and sensitive precise recording of complex molecular spectra. The pulses of a frequency comb excite the sample at regular time intervals. The pulse train of a second comb of different repetition frequency samples the response of the medium, thus producing a time-domain interference pattern, whose Fourier transform yields the spectrum. Access to the fundamental vibrational bands of molecules is still one of the challenges to take up. Despite recent progress [2,5], mid-IR dual-comb spectroscopy is impeded by a lack of convenient comb generators [7]. Raman spectroscopy is an alternative technique for accessing fundamental molecular vibrations that circumvents the need for mid-IR photonics tools.

In this Letter, we extend dual-comb spectroscopy to stimulated Raman scattering. To our knowledge, this is the first report of nonlinear dual-comb spectroscopy. Direct dual-comb emission spectroscopy and optically multiheterodyne-detected femtosecond Raman-induced Kerr-effect (RIKE) spectroscopy are also demonstrated, we believe, for the first time.

In RIKE dual-comb spectroscopy (Fig. 1), a pump picosecond laser and a Stokes femtosecond frequency comb with a repetition frequency of $f_{\text {rep }}$ are spatially and temporally overlapped onto the sample. Photons are transferred from the intense Raman pump laser to the weak Stokes beam when Stokes comb lines coincide with Raman resonances of the sample. The resulting frequency comb with the imprint of stimulated Raman transitions is heterodyned against a frequency comb similar to the Stokes comb, but with a slightly different repetition frequency, $\mathrm{f}_{\text {rep }}+\Delta \mathrm{f}_{\text {rep }}$. The multiheterodyne detection magnifies the weak signal field and downconverts the comb of Raman signals to the rf domain, where it becomes easily accessible to fast digital processing. This scheme without moving parts enables highly multiplexed fast measurements over a spectral span as broad as the bandwidth of the Stokes femtosecond optical comb, with simultaneous access to the real and imaginary parts of the nonlinear susceptibility. Detection of the changes in the polarization of the linearly polarized Stokes comb induced at the sample by the circularly polarized pump, in a scheme similar to femtosecond RIKE spectroscopy [8], provides sensitivity enhancement and reduction of artifacts like cross-phase modulation and parasitic interference patterns.

The experimental setup is displayed in Fig. 2. A femtosecond $\mathrm{Yb}$-fiber-based laser/amplifier system [ $[\underline{\overline{4}}]$ with a repetition frequency of $100 \mathrm{MHz}$ serves as the Raman pump. Its emission is centered at a wavelength of $1.04 \mu \mathrm{m}$, with a full width at half-maximum (FWHM) of $37 \mathrm{~cm}^{-1}$ after spectral filtering, and the pulses are chirped. Therefore, not all spectral components of the pump interact with the probe, leading to an improved spectral resolution. The pump beam is circularly polarized. The Stokes probe laser is a linearly polarized Er-fiber frequency comb emitting about $20 \mathrm{~mW}$ of average power and centered at $1.55 \mu \mathrm{m}$ with an FWHM of $340 \mathrm{~cm}^{-1}$. It is filtered to

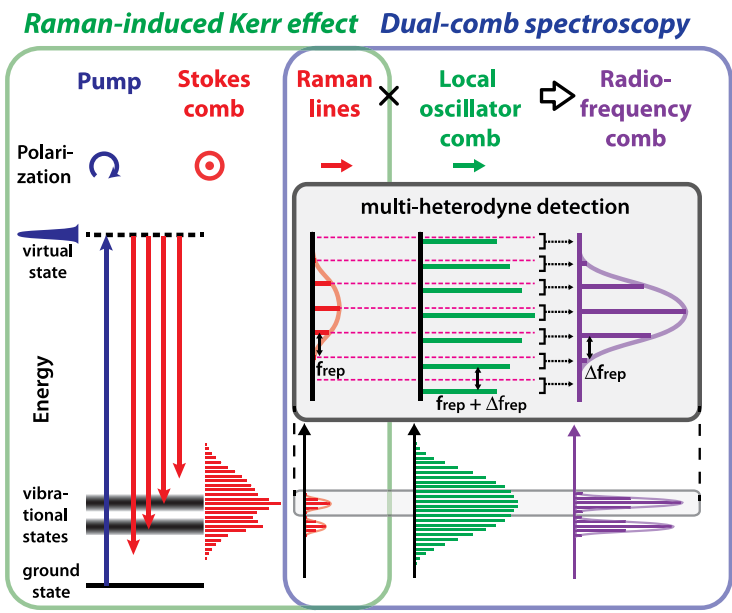

Fig. 1. (Color online) Principle of RIKE dual-comb spectroscopy. 


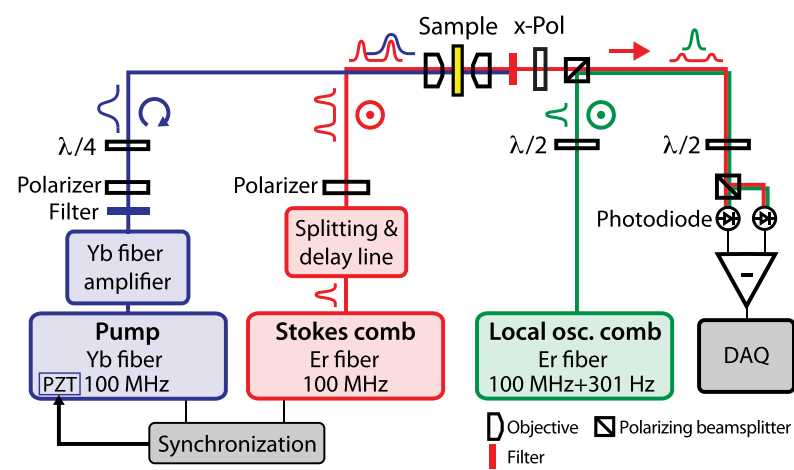

Fig. 2. (Color online) Experimental setup for RIKE dual-comb spectroscopy. x-Pol, crossed-polarizer; $\lambda / 4$, quarter-wave plate; $\lambda / 2$, half-wave plate; PZT, piezo-electric transducer; DAQ, data acquisition.

$150 \mathrm{~cm}^{-1}$ to limit the spectral range in this proof-ofprinciple experiment to the stimulation of Raman signals with shifts around $3000 \mathrm{~cm}^{-1}$, i.e., in the $\mathrm{C}-\mathrm{H}$ stretch region. The repetition frequency of the $\mathrm{Yb}$ pump laser is actively synchronized to the Er comb using a scheme similar to the one reported in [9]. The fundamental repetition frequency and its 100th harmonic are compared to those of the Er-fiber laser. The two resulting error signals are combined through a continuously variable potentiometer and fed back to a piezo-electric transducer inside the Yb oscillator cavity. Sum frequency generation cross-correlation measurements of the spectrally filtered $\mathrm{Yb}$ beam and the Er oscillator output, performed over a time period of a few seconds using two different electronic filtering bandwidths ( $1 \mathrm{MHz}$ and $160 \mathrm{~Hz}$ ), provide a rms timing jitter of 34 and 15 fs, respectively. The output of the erbium oscillator is split to generate a pulse that is temporally overlapped with the Raman pump and a reference pulse used to measure the spectrum of the Stokes laser without pump. The two pulses are separated by about $250 \mathrm{ps}$. An infinity-corrected objective focuses the pump (diameter: $6 \mu \mathrm{m}$, Rayleigh length: $28 \mu \mathrm{m}$ ) and Stokes beams (diameter: $7 \mu \mathrm{m}$, Rayleigh length: $22 \mu \mathrm{m}$ ) into a $1 \mathrm{~mm}$ thick cuvette containing the liquid sample. The positions of the focal points of the pump and Stokes beams are located about $40 \mu \mathrm{m}$ apart due to chromatic aberrations of the microscope objective, and this reduces the efficiency of the stimulated Raman process. At the sample, the pump and Stokes beams have an average power of $960 \mathrm{~mW}$ (energy of $9.6 \mathrm{~nJ}$ ) and $1.9 \mathrm{~mW}$ (energy of $9.5 \mathrm{pJ}$ ), respectively. After recollimation of the beams, the pump light is rejected by a long-wavelength-pass optical filter. A cross-polarizer is aligned to block the Stokes beam in the absence of a pump. The optical birefringence induced in the initially isotropic medium by the anisotropic Raman resonant third-order polarization enables transmission of the background-reduced comb of Raman resonances. The extinction ratio of the Stokes probe through the entire optical setup is $10^{-4}$, mostly limited by the microscope objectives and the sample cell. A second femtosecond Er-fiber comb, with a repetition frequency detuned by $301 \mathrm{~Hz}$ and an average output power of $20 \mathrm{~mW}$, optically samples the trains of pulses transmitted by the polarizer. The amplitude of this local oscillator for multiheterodyne detection is controlled by the combination of a half-wave plate and a polarizing beam splitter to optimize the signal-to-noise ratio in the interferogram. The time-domain interference signal is monitored with a differential InGaAs photodetector, which collects the two outputs of the interferometer. After electronic filtering, the interferometric pattern only contains the Raman gain signal, allowing for efficient amplification. The dynamic range issues associated with dual-comb spectroscopy are therefore partially overcome. Two interferograms, time-delayed by $83 \mu \mathrm{s}$, are sequentially sampled at 100 MSamples/s by a 14 bit data acquisition board. Their complex Fourier transform reveals the phase and amplitude spectra in the presence and absence of the pump laser. Calibration of the wavenumber scale is achieved with the help of an optical spectrum analyzer. The ratio of the spectra with and without pump simultaneously provides the Raman gain spectrum of the dispersive (real part) and resonant (imaginary part) tensor elements of the third-order nonlinear susceptibility, similar to other techniques of heterodyne RIKE [10].

An interferogram is measured within $2.93 \mu \mathrm{s}$, which corresponds to a resolution of $6.7 \mathrm{~cm}^{-1}$ and 23 spectral elements, and the acquisition rate of the interferograms is the difference in repetition frequency of the two erbium lasers, $301 \mathrm{~Hz}$, chosen according to the free spectral range span and the comb line spacing. To get a reasonable signal-to-noise ratio, one hundred interferograms are averaged, resulting in an effective measurement time of $293 \mu \mathrm{s}$ and a total experimental time of $332 \mathrm{~ms}$. Averaging and Fourier transforming may be accomplished in real time with the use of field-programmable gate arrays. Figure 3 displays a portion of the amplitude and phase spectra of neat benzonitrile around $3100 \mathrm{~cm}^{-1}$. The observed linewidth (about $27.5 \mathrm{~cm}^{-1}$ ) of the band is narrower than the width of the Raman pump laser, as the pulses of the latter are chirped. In this spectrum, the stimulated Raman gain experienced by the Stokes beam at the sample is $10^{-5}$ and the relative noise level is $10^{-6}$. The weaker bands of p-Xylene (Fig. 4 ) are recorded with
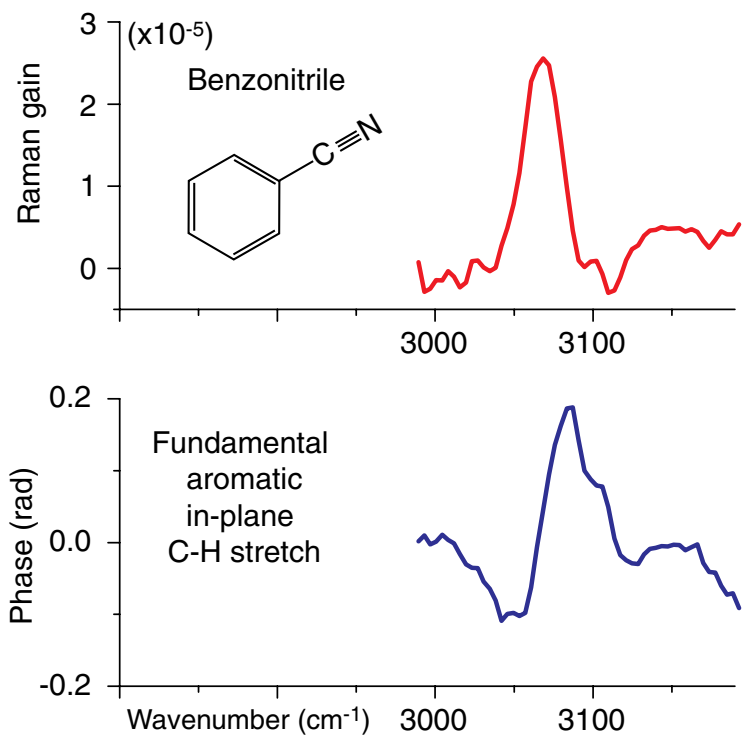

Fig. 3. (Color online) Experimental Raman gain amplitude and dispersion spectra of benzonitrile, measured within $293 \mu \mathrm{s}$. The phase of the pump laser is slowly varying and does not contribute to the resonance in the dispersion spectrum. 


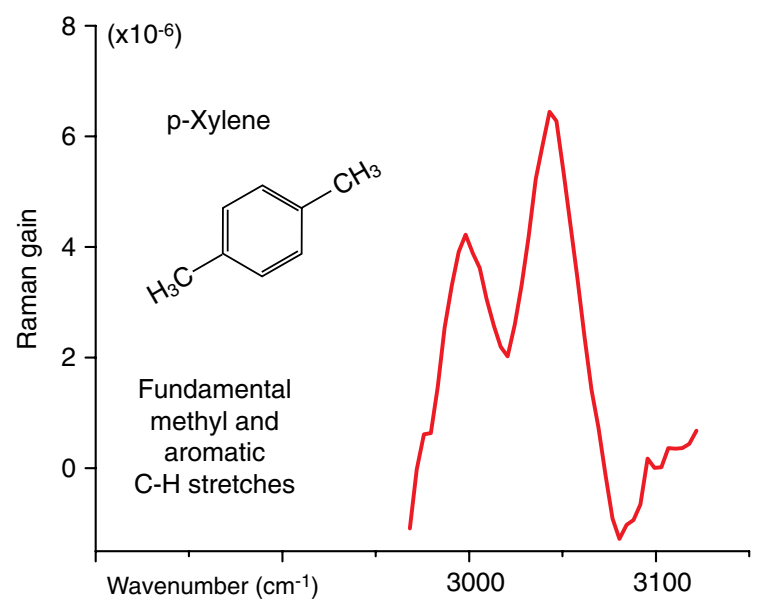

Fig. 4. (Color online) Experimental spectrum of p-Xylene.

the same experimental setup. 1062 interferograms, each measured within $2.99 \mu \mathrm{s}$, are averaged, leading to an effective measurement time of $3.2 \mathrm{~ms}$ and an experimental time of $3.5 \mathrm{~s}$. The noise level in our experiment already represents an excellent figure for real-time femtosecond stimulated Raman spectroscopy. Differential detection of the interferometric signal in the rf domain is one of the causes that lead to such results. Sensitivities better than $10^{-8}$ have been reported in video-rate imaging [11] with lock-in detection, but these only allow for multiplex detection of three spectral elements, whereas RIKE dualcomb spectroscopy potentially allows for broad spans and high resolution. Most femtosecond stimulated Raman experiments (e.g., [8]) use complex amplified laser systems with kilohertz-to-hertz repetition frequencies and long measurement times, although promising alternatives with $\mathrm{MHz}$ systems have already been demonstrated [12]. Our low-intensity setup and our very short measurement time contribute in opening intriguing opportunities for real-time spectrally resolved Raman chemical labeling. Significant sensitivity of our experiment could be achieved through a better spatial overlap between the pump and Stokes beams, either with reflective objectives or with laser systems that emit in spectral regions (e.g., $800 \mathrm{~nm}$ ) where microscopy instrumentation is more advanced.

Much faster acquisition rates may be obtained if the line spacing of the combs matches the desired spectral resolution. Frequency comb generators produced by four-wave mixing in microresonators [13] typically exhibit a line spacing ranging between 10 and $500 \mathrm{GHz}$, and might therefore open up a route to on-chip Raman spectrometers. As the interaction length for the stimulation of Raman transitions is a few tens of micrometers, our instrument suits very well the requirements of microscopic imaging of chemical or biological samples, as well as capillary electrophoresis and microfluidic devices.

Support from the Max Planck Foundation, the European Laboratory for Frequency Comb Spectroscopy, the Munich Center for Advanced Photonics, the European Research Council (Advanced Grant 267854), Eurostars, and the Agence Nationale de la Recherche are acknowledged.

\section{References}

1. T. W. Hänsch, Rev. Mod. Phys. 78, 1297 (2006).

2. E. Baumann, F. R. Giorgetta, W. C. Swann, A. M. Zolot, I. Coddington, and N. R. Newbury, Phys. Rev. A 84, 062513 (2011).

3. T. Ideguchi, A. Poisson, G. Guelachvili, N. Picqué, and T. W. Hänsch, "Adaptive real-time dual-comb spectroscopy," arXiv:1201.4177 (2012).

4. B. Bernhardt, A. Ozawa, P. Jacquet, M. Jacquey, Y. Kobayashi, T. Udem, R. Holzwarth, G. Guelachvili, T. W. Hänsch, and N. Picqué, Nat. Photonics 4, 55 (2010).

5. B. Bernhardt, E. Sorokin, P. Jacquet, R. Thon, T. Becker, I. T. Sorokina, N. Picqué, and T. W. Hänsch, Appl. Phys. B 100, 3 (2010).

6. I. Coddington, W. C. Swann, and N. R. Newbury, Phys. Rev. Lett. 100, 013902 (2008).

7. A. Schliesser, N. Picqué, and T. W. Hänsch, Nat. Photonics 6, 440 (2012).

8. S. Shim and R. A. Mathies, J. Raman Spectrosc. 39, 1526 (2008).

9. D. J. Jones, E. O. Potma, J. Cheng, B. Burfeindt, Y. Pang, J. Ye, and X. S. Xie, Rev. Sci. Instrum. 73, 2843 (2002).

10. C. W. Freudiger, M. B. J. Roeffaers, X. Zhang, B. G. Saar, W. Min, and X. S. Xie, J. Phys. Chem. B 115, 5574 (2011).

11. C. W. Freudiger, W. Min, G. R. Holtom, B. Xu, M. Dantus, and X. S. Xie, Nat. Photonics 5, 103 (2011).

12. E. Ploetz, B. Marx, T. Klein, R. Huber, and P. Gilch, Opt. Express 17, 18612 (2009).

13. C. Wang, T. Herr, P. Del'Haye, A. Schliesser, R. Holzwarth, T. W. Hänsch, N. Picqué, and T. J. Kippenberg, "Midinfrared frequency combs based on crystalline microresonators," arXiv:1109.2716 (2011). 